\title{
Botulinum toxin type A for hand tremor: a meta-analysis of randomised controlled trials
}

\author{
Xiaoyan Zheng, Wenjing Wei, Peidong Liu, Chunxiao Wu, Liming Lu, Chunzhi Tang \\ South China Research Centre for Acupuncture and Moxibustion, Medical College of Acu-Moxi and Rehabilitation, \\ Guangzhou University of Chinese Medicine, Guangzhou, China
}

\begin{abstract}
Background. Tremor is one of the most common movement disorders. It does not usually respond to first-line drug treatments (e.g. propranolol, primidone, anticholinergics, gabapentin and clonazepam) due to side effects and frequent dose limitations. Botulinum toxin type A (BoNT-A) has been widely used to treat tremor, but its efficacy and safety are uncertain.

Aims. To evaluate the efficacy and safety of BoNT-A in the treatment of hand tremor.

Methods. We searched the MEDLINE, EMBASE, PsycINFO and Cochrane Library databases for relevant randomised controlled trials of the effects of BoNT-A injections on tremors, up to 20 February 2020. A meta-analysis of comparative effects was performed using R studio software, and publication bias was examined using Egger's test.

Results. Six studies examining a total of 245 participants with tremor were included in the meta-analysis. The primary outcome of meta-analysis showed no difference in clinical tremor scale scores between the BoNT-A group versus the placebo group (standardised mean difference (SMD): $-0.42,95 \%$ confidence interval $(C I)$ : -1.94 to $1.10 ; l^{2}=96 \%$ ). For clinical tremor scale scores, subgroup analyses suggested that the BoNT-A group may differ in terms of multiple sclerosis (MS) related tremor (SMD: -1.10; $95 \% \mathrm{Cl}:-2.17$ to $-0.04 ; l^{2}=79 \%$ ) compared to a placebo, but the difference did not exist in the outcome of essential tremor (ET) or hand tremor (MD: $\left.-1.31 ; 95 \% \mathrm{Cl}:-3.39 ; 1.31 ; l^{2}=97 \%\right)$. Grip strength (MD: $-1.25,95 \% \mathrm{Cl}:-5.99$ to $3.50, l^{2}=97 \%$ ) was slightly lower in the BoNT-A group, but the difference was not significant. The incidence of adverse events (AEs), including hand weakness (RR: $2.96,95 \% \mathrm{Cl}: 1.40$ to $6.24, P^{2}=37 \%$ ), was significantly greater in the BoNT-A group than in the placebo group. Two studies were assessed as having an overall low risk of bias.
\end{abstract}

Conclusions. Our study confirms that BoNT-A injections are unlikely to have an impact on patients with hand tremors. However, subgroup analysis suggested that BoNT-A injections could have possible benefits in MS-related tremor. While moderate to severe hand weakness AEs often limits their use in clinical practice, additional well-designed double-blind, placebo-controlled trials are needed to provide more robust conclusions.

Key words: systematic review, meta-analysis, botulinum toxin, tremor

(Neurol Neurochir Pol 2020; 54 (6): 561-567)

\section{Introduction}

Tremor is defined as a rhythmic and involuntary movement of any body part [1]. The prevalence of essential tremor (ET) is markedly greater than that of Parkinson's Disease (PD) $[2,3]$, with an overall estimated prevalence of $0.9 \%$ to $2.2 \%$, and $4.6 \%$ of the population aged $\geq 65$ years [4]. PD affects approximately $1 \%$ of the population over the age of 60 , with an incidence of resting or postural tremor [5]. The prevalence of tremor in the multiple sclerosis (MS) population ranges from $25 \%$ to $58 \%$ [6]. Tremor is a symptom of many disorders. In addition to ET [2], PD [5], and MS [6], other conditions also cause tremor, such as dystonic tremor and lesions to the midbrain or cerebellar structures (rubral tremor). Tremor shows a limited response to the pharmacological treatments currently available [7].

Address for correspondence: Liming Lu, Chunzhi Tang, South China Research Centre for Acupuncture and Moxibustion, Medical College of Acu-Moxi and Rehabilitation, Guangzhou University of Chinese Medicine, Guangzhou, China, e-mail: lulimingleon@126.com, jordan664@163.com 
Tremors can be classified as being postural, rest or action tremors. Surgical treatments such as deep brain stimulation (DBS) are effective for a number of movement disorders, including ET, PD tremor and tremor due to MS [8]. For tremor drug options [9], propranolol and primidone are considered the drugs of first choice in ET tremor reduction. Dystonic limb tremor may respond to anticholinergics. Gabapentin and clonazepam are often recommended for orthostatic tremor. MS tremor responds only poorly to drug treatment. For patients with severe MS tremor, thalamic deep brain stimulation has been recommended. Although drug options (e.g. propranolol and primidone) are considered first-line treatments and are effective in the management of ET tremor $[10,11]$, more than $30 \%$ of patients with ET do not respond to these agents. In addition, their use is limited, particularly among people over 70 years old [12].

With pharmacological or surgical management limited due to adverse events and long-term efficacy, an investigation into the effects of alternative treatments for tremor seemed to be worthwhile.

Botulinum toxin type A (BoNT-A) is increasingly often used as a treatment for focal tremors [13], but no meta-analyses of the effects of BONT-A on tremor in patients have been performed. As related clinical research is accumulating, a meta-analysis designed to identify the efficacy and safety of BoNT-A for tremors is timely. This present study aimed to assess the effectiveness and safety of BoNT-A for hand tremors in adults, including patients with ET, PD tremor and MS tremor.

\section{Methods}

$$
\text { Eligibility }
$$

\section{Types of study}

Randomised controlled trials (RCTs) comparing the effects of BoNT-A and control therapy on patients with tremor were evaluated for inclusion, regardless of blinding, language, publication status, and length of trial.

\section{Types of participants}

Study participants were adults (aged 18 or older) with confirmed clinical diagnoses of any type of tremor according to the criteria proposed by the Tremor Investigation Group. Gender or ethnicity restrictions were not applied. According to the principle of PICOS in evidence-based medicine, studies were included if they met the following criteria for participants: all subjects aged $\geq 18$ years with a diagnosis of PD, ET, MS or other tremor disorder. The following were excluded: observational studies, retrospective studies, conference summaries, animal studies, reviews, and duplicate articles.

\section{Types of intervention}

Botulinum toxin injections for tremor were compared to control injections. We did not exclude trials based on the dose or route of administration.

\section{Types of outcome measure}

The primary outcome measures for evaluating the severity of tremors of the limb in patients with ET, PD and MS included the following clinical assessment comparisons: the Fahn-Tolosa-Marin Tremor Rating Scale (FTM-TRS) (range 0-4 points) rates tremor severity based on tremor amplitude from 0 (no tremor) to 4 (severe tremor) in each part of the body; the Essential Tremor Rating Assessment Scale (TETRAS) consists of ten items scoring from $0-4$ points $(0=$ no visible tremor); and the Tremor Clinical Rating Scale (TCRS) has a range of scores from 0 (no tremor) to 4 (severe tremor) points. Secondary outcomes included grip strength (measured by a dynamometer or by a clinical rating of the strength of the wrist flexors and extensors), hand weakness (defined as a percentage decrease in force of the whole hand) as adverse events, kinetic tremor severity (0-4), and postural tremor severity (0-4).

\section{Data source and search strategy}

A search of Medline, CENTRAL (Cochrane Library), PsycINFO and EMBASE databases from their inception until February 2020 was performed to identify potentially qualified studies. Medical Subject Heading (MeSH) terms, key words and text words were used as the search strategy (details are presented in Table S1). This strategy was then adapted to each database, and the search terms were combined using 'AND' or 'OR' operators. We inspected the references of all identified studies to identify additional relevant studies and to avoid omitting important articles.

\section{Selection of reports and data extraction}

Firstly, the reviewer identified duplicate publications using Note Express and sorted duplicate articles into different classifications. An independent reviewer screened the titles and abstracts to determine whether articles met the inclusion criteria using Note Express (first scanning).

\section{Risk-of-bias assessment}

The assessment of the risk of bias in the included studies was based on the recommendations in the methods section of the Cochrane Handbook 5.1.0 [14]. The quality of each included study was independently assessed by XY and PD using the Cochrane Risk of Bias (ROB) tool. The overall risk level for each outcome was marked as low, high, or unclear. Based on these items, studies containing more than half of high-risk or unclear items were considered to be overall high risk; otherwise, they were considered low risk. Two reviewers discussed the results with each other to reach an agreement if a disagreement existed.

\section{Data analysis}

Data was extracted from each study to perform a meta-analysis using $\mathrm{R}$ studio software according to the data type. We obtained $95 \%$ confidence intervals (CIs) and mean 
differences (MDs) to calculate the combined outcomes of continuous variables. The standardised mean difference (SMD) statistic was selected for continuous data when the outcome was assessed by the different scales (e.g. scores of tremor severity scales). Risk ratios (RRs) with $95 \%$ CIs were calculated for dichotomous data (e.g. incidence of adverse events). Differences were estimated to be statistically significant at $p<0.05$. Statistical heterogeneity was calculated with Cochran's $\mathrm{Q}(p<0.1)$ and $\mathrm{I}^{2}$ tests (Higgins et al., 2003). If $p>0.10$ and $\mathrm{I}^{2}<50 \%$, which indicated no homogeneity, a fixed-effects model was used. If $p \leq 0.10$ or $50 \%<\mathrm{I}^{2}<75 \%$, which indicated significant heterogeneity, a random-effects model was used. If $\mathrm{I}^{2}>75 \%$, we either conducted an exclusive sensitivity analysis or abandoned the meta-analysis. For the primary outcome of severity of tremor, we performed the following subgroup analyses: tremor with different causes including PD, ET, MS, and other tremors.

\section{Results}

\section{Study characteristics}

Using the search strategy (Tab. S1), we obtained 1,489 potential studies (MEDLINE database $(n=187)$, EMBASE $(\mathrm{n}=1,228)$, Cochrane Library $(\mathrm{n}=49)$, and PsycINFO $(n=12)$. The search initially identified 1,427 potentially relevant studies. After screening the literature, six RCTs [15-20] were ultimately included (supplementary Figure S1).

We identified six publications reporting on 125 patients, with 98 subjects with tremor receiving BoNT-A injections and 120 subjects receiving a placebo. The tremors were located in the hands of these patients. The intervention duration ranged from 4 weeks to 16 weeks. The toxin was distributed across 7-14 injection sites with an average of nine sites per patient. The total dose of BoNT-A ranged from 50 to 150 international units (IU) per patient. The study characteristics and the consistency evaluation of the extracted data are summarised in supplementary Table S1.

\section{Quality assessment}

Of the six included studies, three $[17,19,20]$ were considered as having a low risk of bias in the domains of random sequence generation, selective reporting, incomplete outcome data and other biases. Only one study [18] was assessed as having a low risk of bias in the domains of blinding of outcome assessment and incomplete outcome data. Two trials were assessed as having an overall low risk of bias. Two reviewers discussed any discrepancies to reach an agreement. The risk of bias summary is presented in Figure 1. A consistency evaluation of the risk-of-bias assessment is presented in supplementary Table S2.

\section{Analysis of outcomes}

\section{Primary outcomes}

\section{Tremor severity}

As shown in Figure 2A, six RCTs reported tremor severity in 125 participants injected with BoNT-A and 120 participants injected with a placebo. The overall pooled results showed a statistically significant reduction in tremor (SMD: -0.42 , $95 \%$ CI: -1.94 to 1.10 ; I2 = 96\%, Fig. 2A).

\section{Subgroup analysis}

Because the pooled results of evaluating tremor severity exhibited high heterogeneity ( $\mathrm{p}<0.01 ; \mathrm{I} 2=96 \%$ ), a subgroup analysis according to types of tremor was conducted to investigate the sources of heterogeneity. Based on different types of tremor, a subgroup analysis was conducted. This revealed a statistically significant reduction in essential hand tremor (SMD: $-1.31,95 \% \mathrm{CI}:-3.93$ to 1.31 , I2 = 97\%, Figure 2B). The subgroup analyses showed that the BoNT-A group differed in terms of MS-related tremor (SMD: -1.10; 95\% CI: -2.17 to $-0.04 ; \mathrm{I} 2=79 \%$, Figure $2 \mathrm{~B}$ ) compared to the placebo. However, no difference existed in the outcome of ET hand tremor.

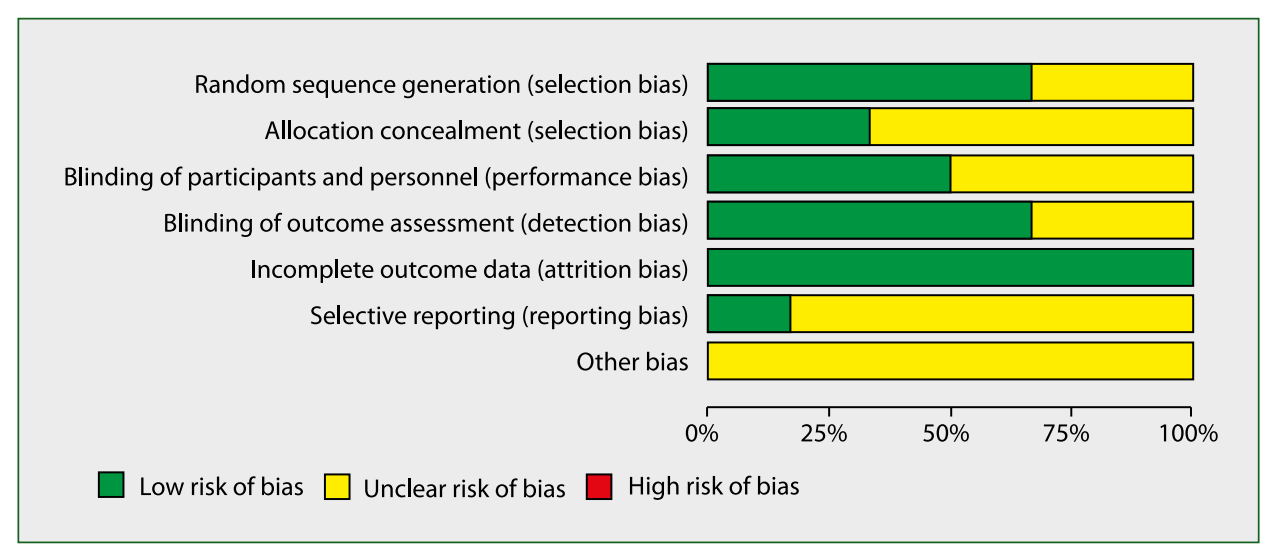

Figure 1. Risk of bias graph 


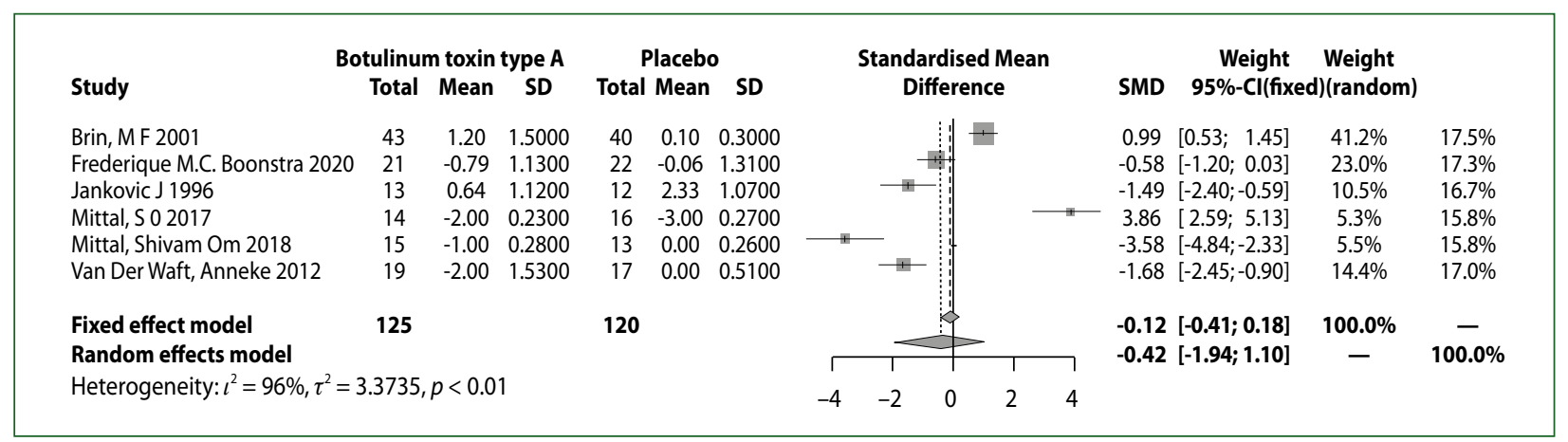

Figure 2A. Forest plot of BoNT-A on tremor severity in meta-analysis. $\mathrm{Cl}$ - confidence interval, SD - standard deviation

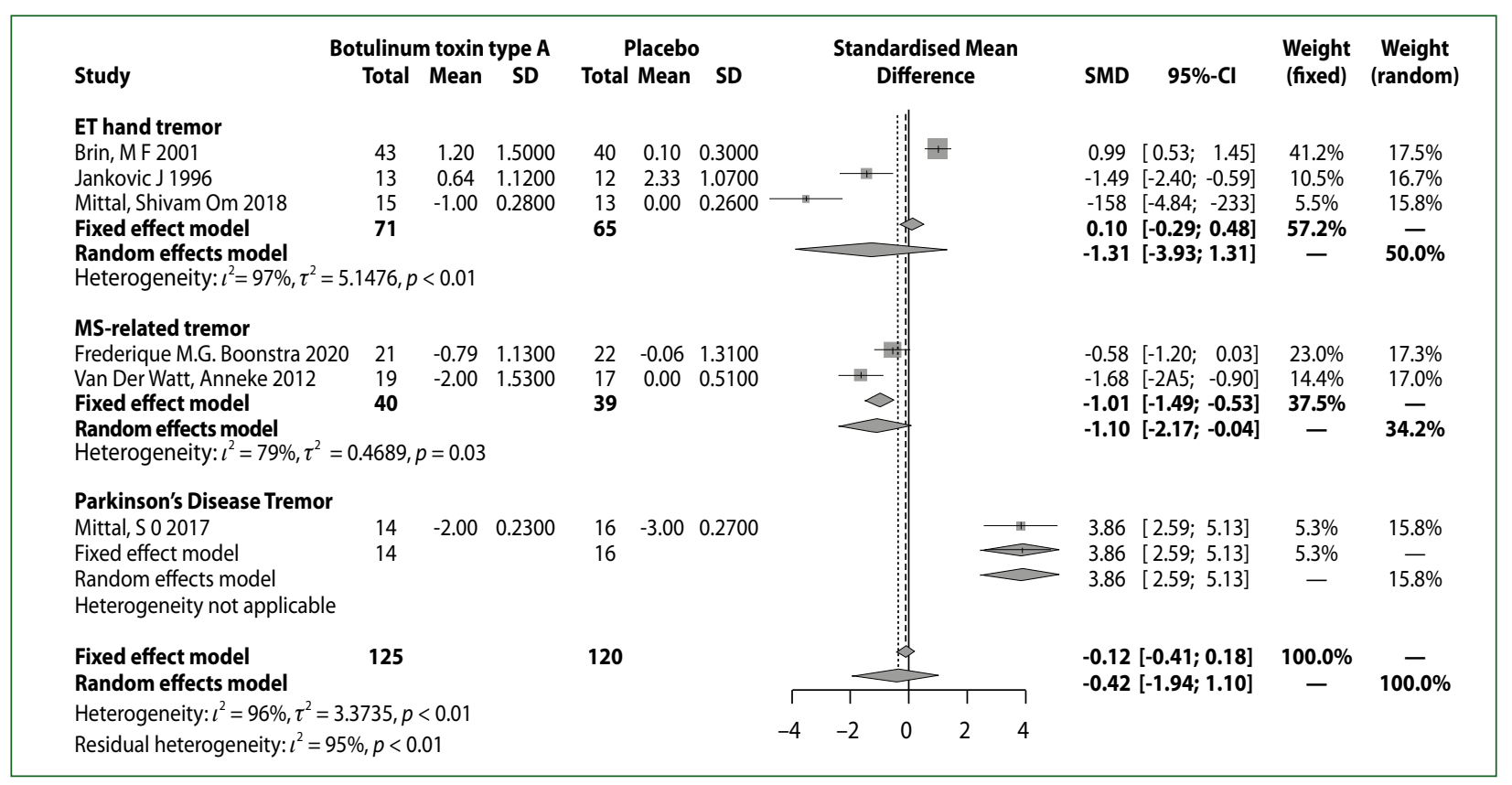

Figure 2B. Subgroup analyses of BoNT-A on tremor severity in meta-analysis. $\mathrm{Cl}$ - confidence interval, IV - inverse variance, SD - standard deviation

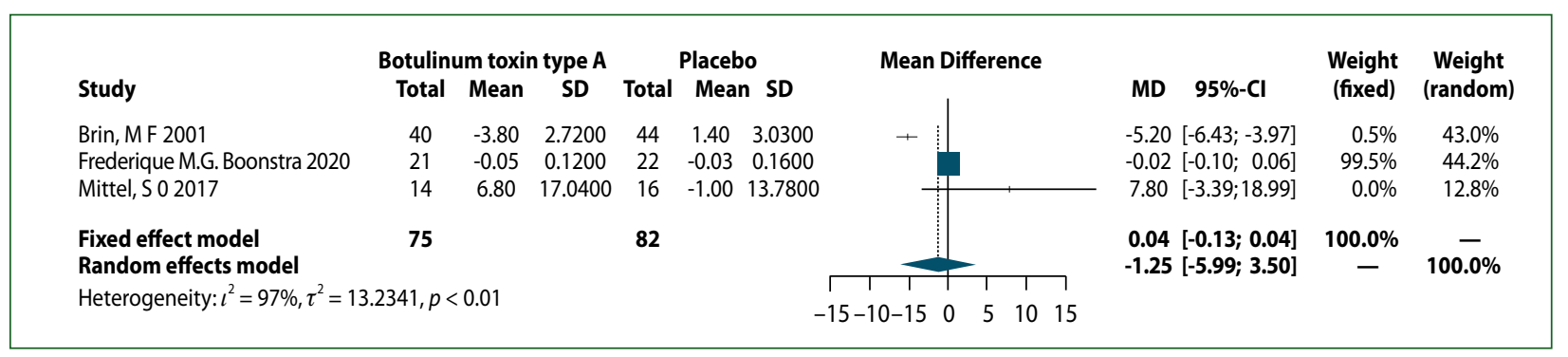

Figure 3. Forest plot of BoNT-A on grip strength in meta-analysis. Cl - confidence interval, SD - standard deviation

\section{Grip strength}

As shown in Figure 3, two RCTs reported grip strength in 75 participants injected with BONT-A and 82 participants injected with a placebo. The overall pooled results did not show a statistically significant improvement in grip strength (MD: $-1.25,95 \%$ CI: -5.99 to 3.50 , I2 = 97\%, Fig. 3).

\section{Hand weakness}

As shown in Figure 4, three RCTs reported an adverse event of hand weakness in 56 participants injected with BoNT-A and in 55 participants injected with a placebo. The overall pooled results showed a statistically significant improvement in hand weakness (RR: 2.96, 95\% CI: 1.40 to 6.24 , 


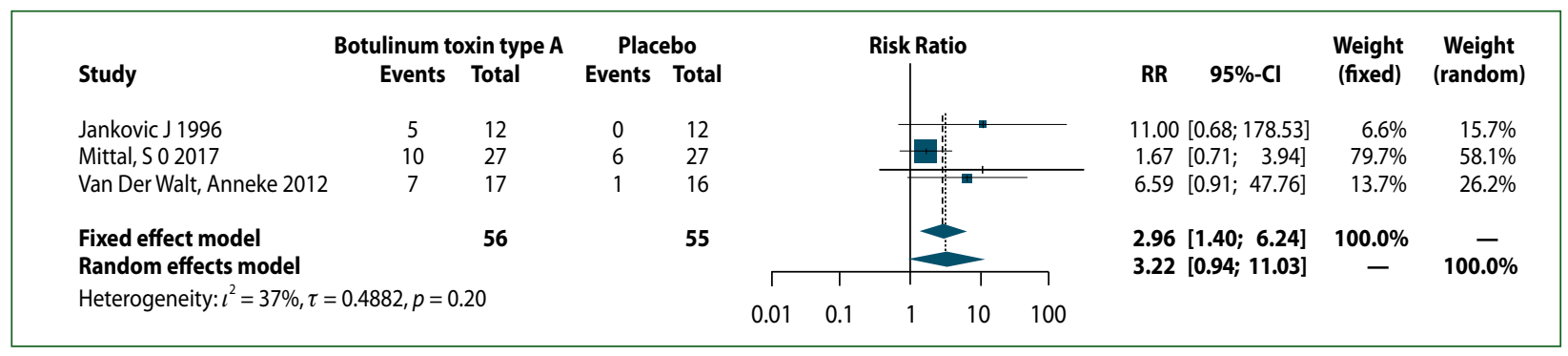

Figure 4. Forest plot of relative risk of BoNT-A on hand weakness in meta-analysis

$\mathrm{I} 2=37 \%$, Fig. 4). BoNT-A injections were effective treatments for patients with hand tremors, but were accompanied by hand weakness, particularly weakness of the finger extensors.

\section{Kinetic tremor severity and postural tremor severity}

Two studies reported a mean change in kinetic tremor severity score from baseline. The pooled results revealed that the BoNT-A groups performed significantly better in terms of kinetic tremor severity (MD: -0.69 ; $95 \%$ CI: -1.28 to -0.11 ; I2 $=98 \%$, supplementary Figure S2) and postural tremor severity (MD: -0.79 ; $95 \% \mathrm{CI}:-1.18$ to -0.40 ; $\mathrm{I} 2=95 \%$, supplementary Figure S3) than the placebo groups.

\section{Publication bias}

A funnel plot is shown in Figure 5. The number of included studies is low, and the available tests for the assessment of publication bias lack statistical power. An Egger's test according to outcomes of tremor severity showed no publication bias of the included studies (Egger's test, $\mathrm{p}=0.71>0.05$ ).

\section{Discussion}

This study included five RCTs comparing the effects of BoNT-A on patients with tremor and included 198 participants. Our study found consistent evidence showing that injections of BoNT-A into the forearm muscles significantly ameliorated tremor, including the severity of upper extremity tremor and grip strength.

These findings support the hypothesis that BoNT-A represents an alternative therapeutic option for patients with tremors who are unresponsive to, or intolerant of, propranolol or primidone. However, adverse effects of BoNT-A were observed, including hand weakness and infrequently pain at the injection site. According to Brin et al. [15], adverse reactions obtained from patient responses to questionnaires throughout the trial showed hand weakness in $30 \%$ (13/43) of the lowdose BoNT-A group and approximately 70\% (31/45) of the high-dose BoNT-A group. As shown in the study by Mittal et al., [19] an injection of IncoA improved ET, with a low occurrence of significant hand weakness. Recent evidence has shown [13] that OnaBoNT-A injections are safe and effective

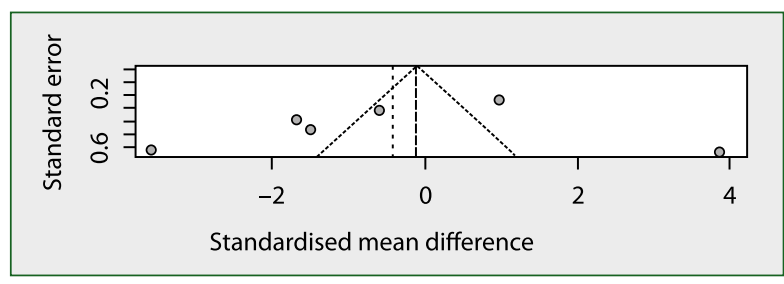

Figure 5. Funnel plot of publication bias of BoNT-A on tremor severity

in the treatment of hand tremor, although the authors did not undertake a meta-analysis.

Between-trial heterogeneity was not observed in the meta-analyses of the primary outcomes, except for the outcome of hand tremor in the ET subgroup. In addition, we did not observe any difference in the outcomes of grip strength in subjects who received the BoNT-A injection based on current evidence, indicating that the BoNT-A injection seems to have little impact on the grip strength of patients with PD, ET and MS.

The effect of BoNT-A on muscle strength is due to a blockade of gamma motor efferents and muscle spindle afferents [21]. All included trials recruited adult patients ( $>18$ years) who had received a clinical diagnosis of $\mathrm{PD}, \mathrm{ET}$, or MS with moderate to severe tremor that was refractory to standard medical treatments. The injection pattern (dose and muscle selection) was customised to each patient and was quantified based on the tremor activity on electromyography (EMG) and the size of the muscle. The number of injection sites in each patient ranged from seven to 14 , with an average of nine sites per patient. The total dose of BoNT-A varied from 50 to 120 units per patient (mean, $100 \mathrm{U}$ per patient). In the placebo group ( $0.9 \%$ saline), the same techniques were utilised to mask the active treatment. Doses of botulinum toxin are usually divided into several injections, depending on the size of the muscle, with up to eight injection sites reported for each muscle [22]. This personalised muscle selection and toxin dose successfully ameliorated tremors [23] and reduced the dose-dependent adverse reactions (e.g. finger weakness and pain at the injection sites).

Regarding the quality of the evidence, key sources of bias exist in these studies and included the limited allocation concealment to the treatment group and the blinding of participants. 
Well-designed and implemented RCTs are the gold standard for evaluating the efficiency of interventions and providing outcome estimates and confidence [23, 24]. Based on our findings, the reporting quality of RCTs assessing the effects of BoNT-A on tremor is unsatisfactory. We searched the Clinicaltrials.gov website and identified many high-quality ongoing studies. We look forward to the publication of these high-quality studies designed to evaluate the tolerance of botulinum toxin injections as a treatment for tremor in the future.

\section{Recommendations for practice}

Regarding the clinical implications, the administration of BoNT-A via a fixed injection approach improves the clinical rating score of tremors, but a high percentage of patients (30-70\%) develop moderate to severe hand weakness, which has limited its use in clinical practice. In a qualitative summary of the included evidence, the injection pattern (dose and muscle selection) ranged from seven to 14 sites, with an average of nine sites per patient, and the total dose ranged from 50 to 120 units per patient (mean, $100 \mathrm{U}$ per patient). We recommend customising the injection pattern for each patient based on the tremor activity on electromyography (EMG) and the size of the muscle.

\section{Limitations}

Some limitations exist in the present study. Firstly, most of the included studies were pilot studies, and only five RCTs were included. Limited evidence of the effectiveness of BoNT-A to improve tremor symptoms is available, and larger randomised trials are required. Secondly, the number of patients included in the meta-analysis of the primary outcome was small, which may lead to false-negative results due to insufficient statistical power. Thirdly, heterogeneity in some domains of the risk of bias resulted in methodological limitations of the included studies. We are hopeful that more powerful evidence will be obtained to provide a more reliable conclusion.

We have provided a meta-analysis of all RCTs where a form of botulinum toxin was used to treat arm tremor. Limited by the small number of studies that could be included, the heterogeneity of tremor across the different neurological conditions under study would further make it difficult to make any disease-specific recommendation.

\section{Conclusions}

BoNT-A injections are ineffective in patients with hand tremors among a mixed population of patients diagnosed with PD, ET and MS, although subgroup analysis suggested that BoNT-A injections has possible benefits in MS-related tremor. Larger randomised trials are required to confirm the clinical effectiveness of BoNT-A in improving tremor. More research is needed to monitor the adverse events associated with this treatment.
Competing interests: The authors have no competing interest nor any conflict of interest to declare.

Acknowledgements: This study was supported by the following grant: NSFC/81873375 and Scientific Research Team Training Project of GZUCM (No.: 2019KYTD203) to Chunzhi Tang Contributions: Xiaoyan Zheng, Chunzhi Tang and Liming Lu conceived and designed the study. Xiaoyan Zheng, Peidong Liu and Wenjing Wei selected the articles and extracted the data. The risk-of-bias assessment was performed independently by Xiaoyan Zheng and Peidong Liu. Xiaoyan Zheng and Wenjing Wei analysed the data. Xiaoyan Zheng wrote the first draft of the manuscript. Chunzhi Tang and Liming Lu interpreted the data and wrote the final version. All authors read and met the ICMJE criteria for authorship, and agree with the results and conclusions of this article.

\section{References:}

1. Elias WJ, Shah BB. Tremor. JAMA. 2014; 311(9): 948-954, doi: 10.1001/jama.2014.1397, indexed in Pubmed: 24595779.

2. Liu HC, Wang SJ, Fuh JL, et al. The Kinmen Neurological Disorders Survey (KINDS): A Study of a Chinese Population. Neuroepidemiology. 1997; 16(2): 60-68, doi: 10.1159/000109672.

3. Larsson T. Sjogren T, Essential tremor: a clinical and genetic population study. Acta Psychiatr Scand Suppl, 1960. 36(144): p. : 1-176.

4. Louis ED, Ferreira JJ. How common is the most common adult movement disorder? Update on the worldwide prevalence of essential tremor. Mov Disord. 2010; 25(5): 534-541, doi: 10.1002/mds.22838, indexed in Pubmed: 20175185.

5. Pringsheim, T., , The prevalence of Parkinson's disease: a systematic review and meta-analysis. Mov Disord, 2014. 29(13): p. : 1583-90.

6. Labiano-Fontcuberta A, Benito-León J. Understanding Tremor in Multiple Sclerosis: Prevalence, Pathological Anatomy, and Pharmacological and Surgical Approaches to Treatment. Tremor and Other Hyperkinetic Movements. 2012; 2(0): 02, doi: 10.5334/tohm.109.

7. Lyons KE, Pahwa R. Pharmacotherapy of essential tremor : an overview of existing and upcoming agents. CNS Drugs. 2008; 22(12): 1037-1045, doi: 10.2165/0023210-200822120-00006, indexed in Pubmed: 18998741.

8. Baizabal-Carvallo JF, Jankovic J. Movement disorders induced by deep brain stimulation. Parkinsonism Relat Disord. 2016; 25: 1-9, doi: 10.1016/j.parkreldis.2016.01.014, indexed in Pubmed: 26806438.

9. Schneider SA, DeuschI G. The treatment of tremor. Neurotherapeutics. 2014; 11(1): 128-138, doi: 10.1007/s13311-013-0230-5, indexed in Pubmed: 24142589.

10. Koller WC, Vetere-Overfield B. Acute and chronic effects of propranolol and primidone in essential tremor. Neurology. 1989; 39(12): $1587-$ 1588, doi: 10.1212/wnl.39.12.1587, indexed in Pubmed: 2586774.

11. Wasielewski PG, Burns JM, Koller WC. Pharmacologic treatment of tremor. Mov Disord. 1998; 13 Suppl 3: 90-100, doi: 10.1002/ mds.870131316, indexed in Pubmed: 9827602.

12. Atuah, K.N., D. Hughes and M. Pirmohamed, Clinical pharmacology: special safety considerations in drug development and pharmacovigilance. Drug Saf, 2004. 27(8): p. : 535-54.

13. Niemann N, Jankovic J. Botulinum Toxin for the Treatment of Hand Tremor. Toxins (Basel). 2018; 10(7), doi: 10.3390/toxins10070299, indexed in Pubmed: 30029483. 
14. Savović J, Weeks L, Sterne JAC, et al. Evaluation of the Cochrane Collaboration's tool for assessing the risk of bias in randomized trials: focus groups, online survey, proposed recommendations and their implementation. Syst Rev. 2014; 3: 37, doi: 10.1186/2046-4053-337, indexed in Pubmed: 24731537.

15. Brin MF, Lyons KE, Doucette J, et al. A randomized, double masked, controlled trial of botulinum toxin type A in essential hand tremor. Neurology. 2001; 56(11): 1523-1528, doi: 10.1212/wnl.56.11.1523, indexed in Pubmed: 11402109.

16. Jankovic J, Schwartz K, Clemence W, et al. A randomized, double-blind, placebo-controlled study to evaluate botulinum toxin type A in essential hand tremor. Mov Disord. 1996; 11(3): 250-256, doi: 10.1002/mds.870110306, indexed in Pubmed: 8723140.

17. Mittal S, Machado D, Richardson D, et al. Botulinum Toxin in Parkinson Disease Tremor. Mayo Clinic Proceedings. 2017; 92(9): 13591367, doi: 10.1016/j.mayocp.2017.06.010.

18. Van Der Walt A, Sung S, Spelman T, et al. A double-blind, randomized, controlled study of botulinum toxin type A in MS-related tremor. Neurology. 2012; 79(1): 92-99, doi: 10.1212/WNL.0b013e31825dcdd9, indexed in Pubmed: 22753445.

19. Mittal SOm, Machado D, Richardson D, et al. Botulinum toxin in essential hand tremor - A randomized double-blind placebo-controlled study with customized injection approach. Parkinsonism Relat Disord. 2018; 56: 65-69, doi: 10.1016/j.parkreldis.2018.06.019, indexed in Pubmed: 29929813.

20. Boonstra FMC, Evans A, Noffs G, et al. OnabotulinumtoxinA treatment for MS-tremor modifies fMRI tremor response in central sensory-motor integration areas. Mult Scler Relat Disord. 2020; 40: 101984, doi: 10.1016/j.msard.2020.101984, indexed in Pubmed: 32062446.

21. Ferreira J, Costa J, Coelho M, et al. The management of cervical dystonia. Expert Opinion on Pharmacotherapy. 2007; 8(2): 129-140, doi: 10.1517/14656566.8.2.129.

22. Zakin E, Simpson D. Botulinum Toxin in Management of Limb Tremor. Toxins (Basel). 2017; 9(11), doi: 10.3390/toxins9110365, indexed in Pubmed: 29125566.

23. Safarpour Y, Jabbari B. Botulinum Toxin Treatment of Movement Disorders. Curr Treat Options Neurol. 2018; 20(2): 4, doi: 10.1007/ s11940-018-0488-3, indexed in Pubmed: 29478149.

24. Wood L, Egger M, Gluud LL, et al. Empirical evidence of bias in treatment effect estimates in controlled trials with different interventions and outcomes: meta-epidemiological study. BMJ. 2008; 336(7644): 601-605, doi: 10.1136/bmj.39465.451748.AD, indexed in Pubmed: 18316340. 